\title{
Analytical procedure for the determination of tetracyclines in medicated feedingstuffs by liquid chromatography-mass spectrometry
}

\author{
Ewelina Patyra, Krzysztof Kwiatek \\ Department of Hygiene of Animal Feedingstuffs \\ National Veterinary Research Institute, 24-100 Pulawy, Poland \\ ewelina.patyra@piwet.pulawy.pl
}

Received: October 26, $2015 \quad$ Accepted: March 10, 2016

\begin{abstract}
Introduction: The article presents a rapid and simple analytical procedure for determination of four tetracyclines (oxytetracycline, tetracycline, chlortetracycline, and doxycycline) in animal medicated feedingstuffs. Material and Methods: Two-gramme samples were extracted by a Na2EDTA-McIlvaine buffer ( $\mathrm{pH} 4$ )/methanol mixtures (40/60, v/v). The determination was achieved by liquid chromatography using a Zorbax Eclipse XDB C18 analytical column with mass spectrometer detection (LC-MS). Results: Recoveries of the antibiotics from spiked feed samples ranged from $78.2 \%$ to $113.5 \%$. The LOD and LOQ for tetracyclines in feeds ranged from 2.8 to 4.2 and from 4.3 to $5.7 \mathrm{mg} / \mathrm{kg}$, respectively. Conclusion: The method was successfully validated and proved to be efficient, precise, and useful for quantification of tetracyclines in medicated feedingstuffs.
\end{abstract}

Keywords: feeds, tetracyclines, LC-MS, solid-liquid extraction.

\section{Introduction}

Tetracyclines (TCs) produced by Streptomyces spp. are broad-spectrum agents, exhibiting activity against wide range of Gram-positive and Gram-negative bacteria, protozoan parasites, and atypical bacteria (chlamydiae, rickettsiae, and mycoplasmas). The members of the TCs group include tetracycline (TC), chlortetracycline (CTC), oxytetracycline (OTC), and doxycycline (DC) (7). These antibiotics are used for the treatment of primary and secondary infections of the digestive and urinary tracts, and respiratory infections in turkeys, chickens, pigs, and ducks. Because of the broadspectrum activity and low production costs (21), the TCs group accounts for over $40 \%$ of all antibiotics used in veterinary medicine in Poland.

Since 2006, when the European Commission banned the use of antibiotic growth promoters, the demand for production of medicated feedingstuffs has been observed. However, overuse of antibacterial drugs in animal production may result in the development of resistance of microorganisms, allergic reactions or toxic effects, and the presence of antibiotic residues in the edible tissues, milk, and/or eggs.
There are several analytical techniques used to quantify TCs in feeds: TLC (15), $\operatorname{HPLC}(4,11,16,17$, 20), HPCE $(11,22), \mathrm{LC} / \mathrm{MS}, \mathrm{LC}-\mathrm{MS} / \mathrm{MS}(6,8,10)$, and microbiological assays (15). Nowadays, liquidchromatography coupled with mass spectrometry and tandem mass spectrometry seem to be the techniques of choice for analysis of these groups of antibiotics. The advantage of these methods is their good sensitivity and capability of identification of target analytes.

Feeds are very complex and have variable matrixes, and some feed components such as lipids, proteins, vegetable oils, and others components which can be co-extracted with the analytes may disturb the analysis. Moreover, tetracyclines are subject to chelation with the transition metal ions, such as $\mathrm{Fe}^{2+}$, $\mathrm{Fe}^{3+}, \mathrm{Al}^{3+}, \mathrm{Cr}^{3+}, \mathrm{Mn}^{2+}, \mathrm{Co}^{2+}, \mathrm{Ni}^{2+}, \mathrm{Cu}^{2+}, \mathrm{Zn}^{2+}, \mathrm{Cd}^{2+}$, $\mathrm{Hg}^{2+}$, and salts with alkali and alkaline earth metals and organic and inorganic acids (e.g. citric, boric, and humic acids). Therefore, for the extraction of this group of compounds the McIlvaine buffer containing EDTA is the most widely used solution, due to its properties of complexing metal cations (4).

So far, there has been a relatively limited number of studies devoted to the determination of the presence 
of tetracycline antibiotics in feeds. Ethyl acetate, methanol mixtures of methanol with water or citric acid, citric acid with acetonitrile or formic acid, and phosphate buffer solution in the presence of trichloroacetic acid were used for the extraction of tetracycline antibiotics from feeds (23). The additional cleaning and preconcentration of the extracts are usually carried out by SPE with the use of an HLB, BondElut C18, or a Varian MP1 cartridges (14).

The aim of this study was to develop a method for simultaneous determination of selected tetracycline antibiotics, which are widely used in veterinary medicine, in medicated feedingstuffs. The method involved a rapid and simple extraction procedure and determination of tetracyclines in feeds by liquid chromatography coupled with mass spectrometer with a single quadrupole.

\section{Material and Methods}

Reagents and chemicals. Tetracycline hydrochloride, oxytetracycline hydrochloride, chlortetracycline hydrochloride, and doxycycline hyclate were purchased from Sigma-Aldrich (USA). All standard solutions were prepared in HPLC-grade methanol from Merck (Germany). Formic acid, acetic acid, and ethylenediaminetetraacetic acid disodium salt dehydrate ( $\mathrm{Na}_{2}$ EDTA) were from Sigma-Aldrich (USA), citric acid was from Acros Organics (USA), and HPLC-grade acetonitrile was from Merck (Germany). Water was purified using Milli-Q water generated by a Milli-Q Plus Water Purification System (Millipore, USA).

Instrumentation. LC-MS analysis was carried out on HP 1200 Series Agilent Technologies (USA) liquid chromatograph with Agilent 6140 quadrupole mass spectrometer (Agilent Technologies, USA). The mass spectrometer was operated in electrospray positive ionisation mode (ESI+). The following mass spectrometer parameters were used: capillary voltage $2000 \mathrm{~V}$, drying gas temperature $-350^{\circ} \mathrm{C}$, drying gas flow - $13 \mathrm{~L} / \mathrm{min}$, and nebulising gas pressure $-40 \mathrm{psi}$. Molecular masses of the precursor ions of OTC, TC, CTC, and DC were 461, 445, 479, and $445 \mathrm{~m} / \mathrm{z}$ respectively.

Three different chromatographic columns: Zorbax XDB C18 $(150 \times 4.6 \mathrm{~mm}, 5 \mu \mathrm{m}) \quad$ (Agilent Technologies, USA), Luna C18 $(150 \times 4.6 \mathrm{~mm}, 5 \mu \mathrm{m})$ (Phenomenex, USA), and Kinetex C18 $(100 \times 2.6 \mathrm{~mm}$, $5 \mu \mathrm{m})$ (Phenomenex USA) were used.

Chromatographic conditions. LC-MS separation of tetracyclines was accomplished with gradient elution on Zorbax Eclipse XDB C18 column $(150 \times 4.6 \mathrm{~mm}$, $5 \mu \mathrm{m}$ ) (Agilent Technologies, USA). Mobile phase consisted of $0.1 \%$ formic acid in ultrapure water (eluent A) and $0.1 \%$ formic acid in acetonitrile (v/v) (eluent B). The gradient was as follows: initial $22 \%$ A increased linearly to $32 \%$ for $11 \mathrm{~min}$ and held for $1 \mathrm{~min}$. Finally, the gradient was returned to the initial $22 \%$ A for $3 \mathrm{~min}$. The column thermostat was set at $30^{\circ} \mathrm{C}$ and the flow rate was $0.6 \mathrm{~mL} / \mathrm{min}$. The injection volume was $3 \mu \mathrm{L}$ and all compounds were eluted within 15 min.

Standard solution. The stock standard solutions of CTC, DC, OTC, and TC were prepared by weighing $50 \pm 0.1 \mathrm{mg}$ of standard substances and dissolving them in $5 \mathrm{~mL}$ of methanol. The solution was stable for six months and stored at $-18^{\circ} \mathrm{C}$.

Extraction and clean-up. Feed samples were ground and then $2 \mathrm{~g}$ were weighed into $200 \mathrm{~mL}$ Erlenmeyer flask. Next $10 \mathrm{~mL}$ of $4: 6(\mathrm{v} / \mathrm{v})$ McIlvaine buffer-Na $\mathrm{N}_{2}$ EDTA ( $\mathrm{pH}$ 4): methanol was added. The samples were shaken for $30 \mathrm{~min}$ on a horizontal shaker and centrifuged for $20 \mathrm{~min}$ at $4000 \mathrm{rpm}$. One hundred microlitres of the supernatant was 100 -fold diluted in deionised water, vortexed for $1 \mathrm{~min}$, and injected into the chromatographic system.

Validation procedure. The analytical procedure was evaluated for specificity, linearity, repeatability, and reproducibility. To determine the specificity of the method, blank samples of feedingstuff were analysed. Limit of detection (LOD) and limit of quantification (LOQ) were calculated on the basis of signal to noise ratio and were $\mathrm{S} / \mathrm{N}=3$ for $\mathrm{LOD}$ and $\mathrm{S} / \mathrm{N}=10$ for $\mathrm{LOQ}$. Two grammes of a drug-free feedingstuff sample were spiked with drug solution to obtain desired concentrations. The calibration curves for standards solutions of CTC, TC, OTC, and DC with five calibration levels $0.1,0.25,0.5,1$, and $3 \mu \mathrm{g} / \mathrm{mL}$ were prepared and the parameters of linear regression were estimated. Finally, these solutions were analysed by LC-MS and calibration curve was plotted.

The repeatability, reproducibility, and recovery were determined by spiking blank feedingstuff samples at three different concentrations: 50, 500, and 1500 $\mathrm{mg} / \mathrm{kg}$, which corresponded to $0.1,0.5$, and $3 \mu \mathrm{g} / \mathrm{mL}$.

\section{Results}

The developed procedure was designed to obtain a qualitative method of determination of the presence of tetracyclines in medicated feedingstuffs.

ESI-MS conditions were optimised by direct infusion of a standard solution of $1 \mu \mathrm{g} / \mathrm{mL}$ of each analyte at a flow rate $0.1 \mathrm{~mL} / \mathrm{min}$. Standard solutions of analytes were prepared in a mixture of methanol and water in the ratio $1: 1(\mathrm{v} / \mathrm{v})$ and injected into the ESI source in positive mode. Under the experimental conditions, full scan provides protonated ions $\left([\mathrm{M}+\mathrm{H}]^{+}\right)$ for each analyte. For CTC and OTC, molecular ions $\mathrm{m} / \mathrm{z} 479$ and 461 were monitored. For TC and DC, the molecular ion $\mathrm{m} / \mathrm{z} 445$ was monitored. Other parameters, such as drying gas flow, temperature of drying gas, nebuliser pressure, capillary voltage, 
fragmentor, and ions monitored were studied, selecting the optimum conditions indicated in section material and methods.

Gradient elution utilised three different octadecyl chromatographic columns: Zorbax Eclipse XDB C18 column $(150 \times 4.6 \mathrm{~mm}, 5 \mu \mathrm{m})$, Thermo BDS C18 $(150 \times 4.6 \mathrm{~mm}, 5 \mu \mathrm{m})$, and Kinetex C18 $(100 \times$ $4.6 \mathrm{~mm}, 2.6 \mu \mathrm{m})$. The best separation results were achieved on a Zorbax Eclipse XDB C18 column. The results of separation of TCs for three test columns are presented in Figs 1, 2, and 3.

In this study, three different mobile phases were compared: the first consisting of $1 \%$ acetic acid and acetonitrile, the second comprising $0.1 \%$ formic acid and acetonitrile, and the third mobile phase consisting of $0.1 \%$ formic acid and $0.1 \%$ formic acid in acetonitrile. The mobile phases were tested on a Zorbax Eclipse XDB C18 column. The results showed that the best separation of TCs and symmetrical peaks were obtained for a mobile phase consisting of $0.1 \%$ formic acid and $0.1 \%$ formic acid in acetonitrile. Representative chromatograms are showed in Figs 4, 5 , and 6 .

The results of the method validation are shown in Tables 1 and 2 . The linearity was evaluated by fivepoint calibration curve with triple analysis. The high correlation coefficients $\left(\mathrm{r}^{2}>0.99\right)$ indicated good correlations between analyte concentrations and peak areas from $50-1500 \mathrm{mg} / \mathrm{kg}$ for the LC-ESI-MS analyses.

Recoveries and precision assay of the proposed method were evaluated using spiked feed samples at the levels of 50,500 , and $1500 \mathrm{mg} / \mathrm{kg}$. The relative OTC, TC, CTC, and DC recoveries for LC-MS method ranged from $91.3 \%$ to $113.5 \%$, from $82.0 \%$ to $96.1 \%$, from $78.2 \%$ to $82.8 \%$, and from $84.8 \%$ to $101.0 \%$, respectively.

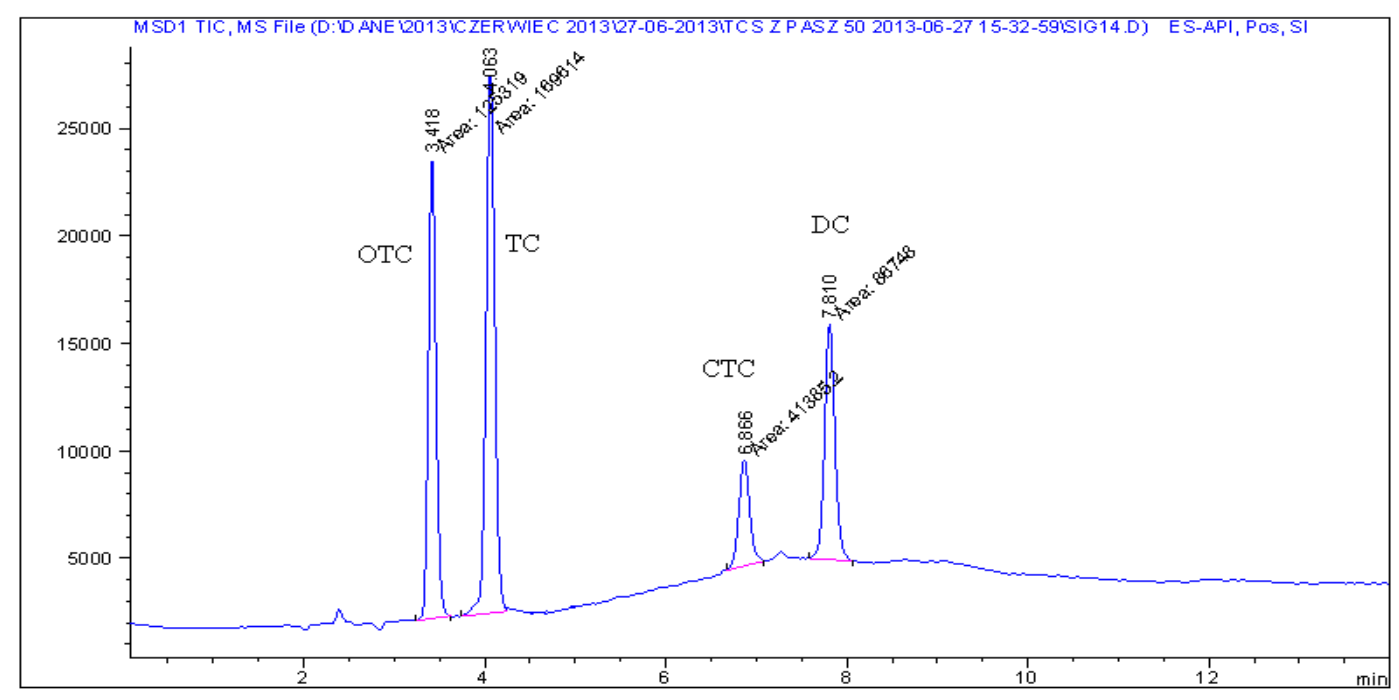

Fig. 1. HPLC chromatogram of a mixture of four TCs $(0.1 \mu \mathrm{g} / \mathrm{mL})$ on a Zorbax Eclipse XDB C18 column

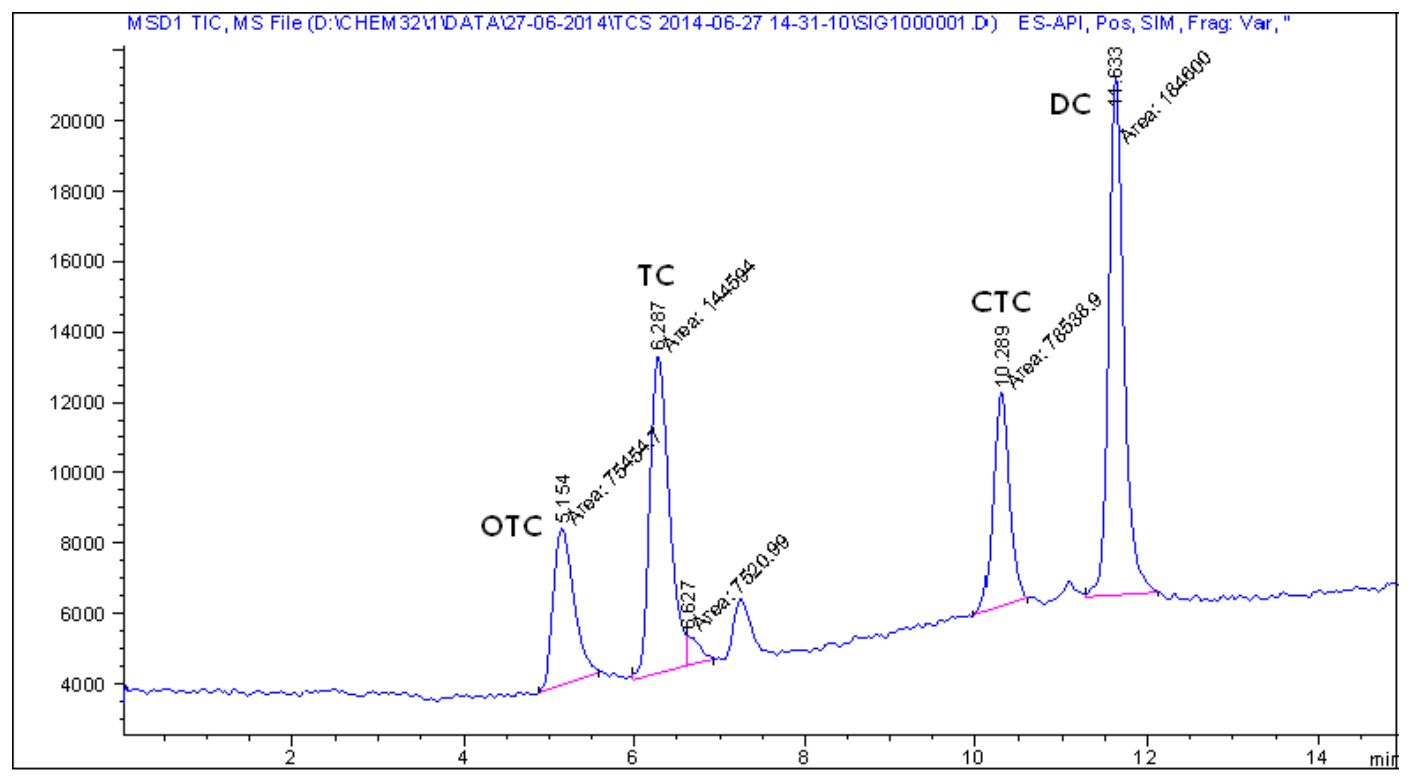

Fig. 2. HPLC chromatogram of a mixture of four TCs $(0.1 \mu \mathrm{g} / \mathrm{mL})$ on a Thermo BDS C18 column 


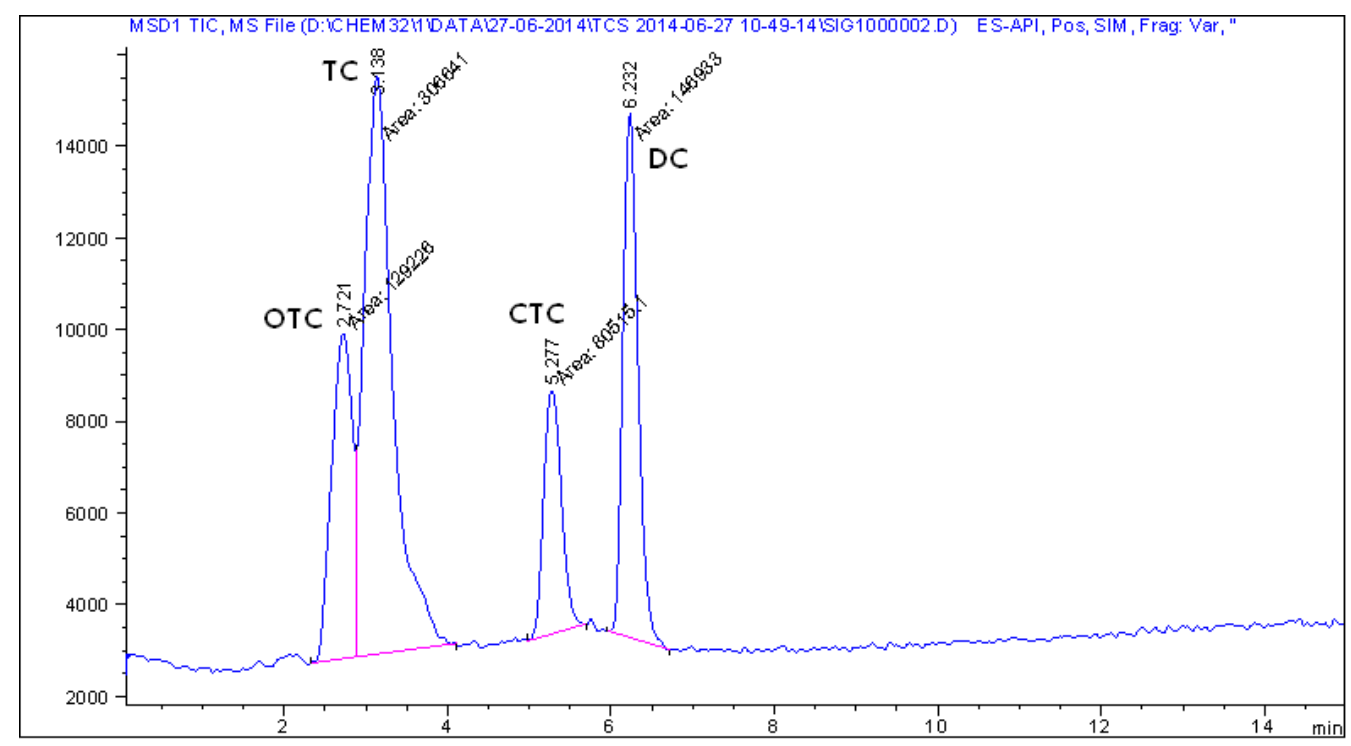

Fig. 3. HPLC chromatogram of a mixture of four TCs $(0.1 \mu \mathrm{g} / \mathrm{mL})$ on a Kinetex $\mathrm{C} 18$ column

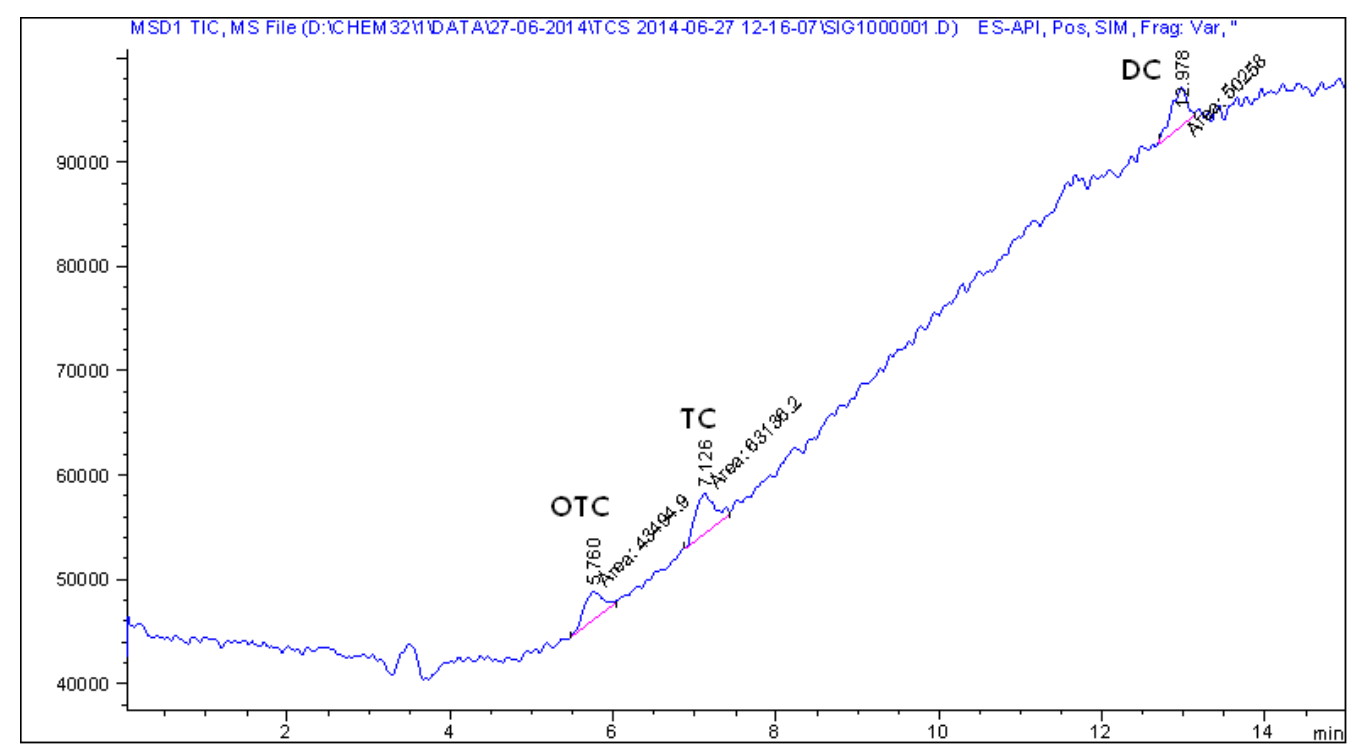

Fig. 4. LC-MS chromatogram of OTC, TC, CTC, and DC on a Zorbax Eclipse XDB C18 column with a mobile phase: $\mathrm{ACN} / 1 \%$ acetic acid in water (no peak of CTC)

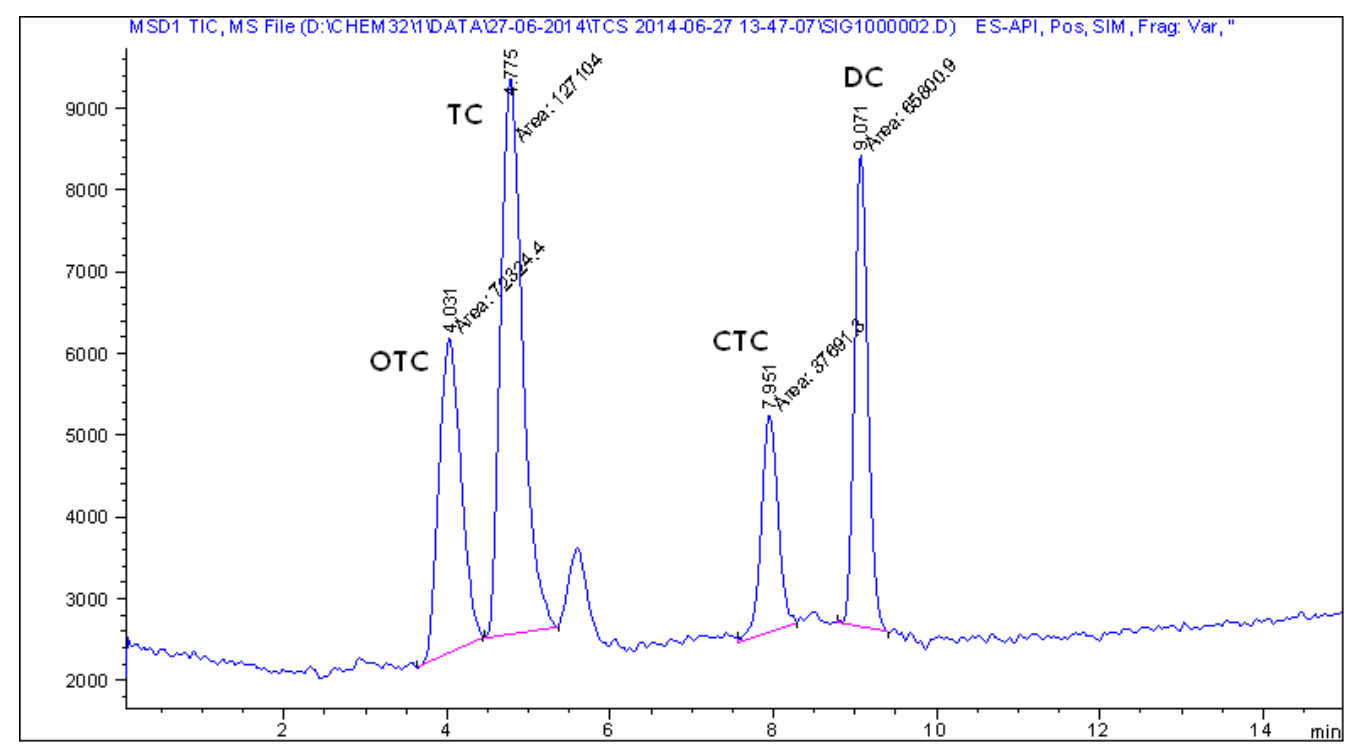

Fig. 5. LC-MS chromatogram of OTC, TC, CTC, and DC on a Zorbax Eclipse XDB C18 column with a mobile phase: $\mathrm{ACN} / 0.1 \%$ formic acid in water 


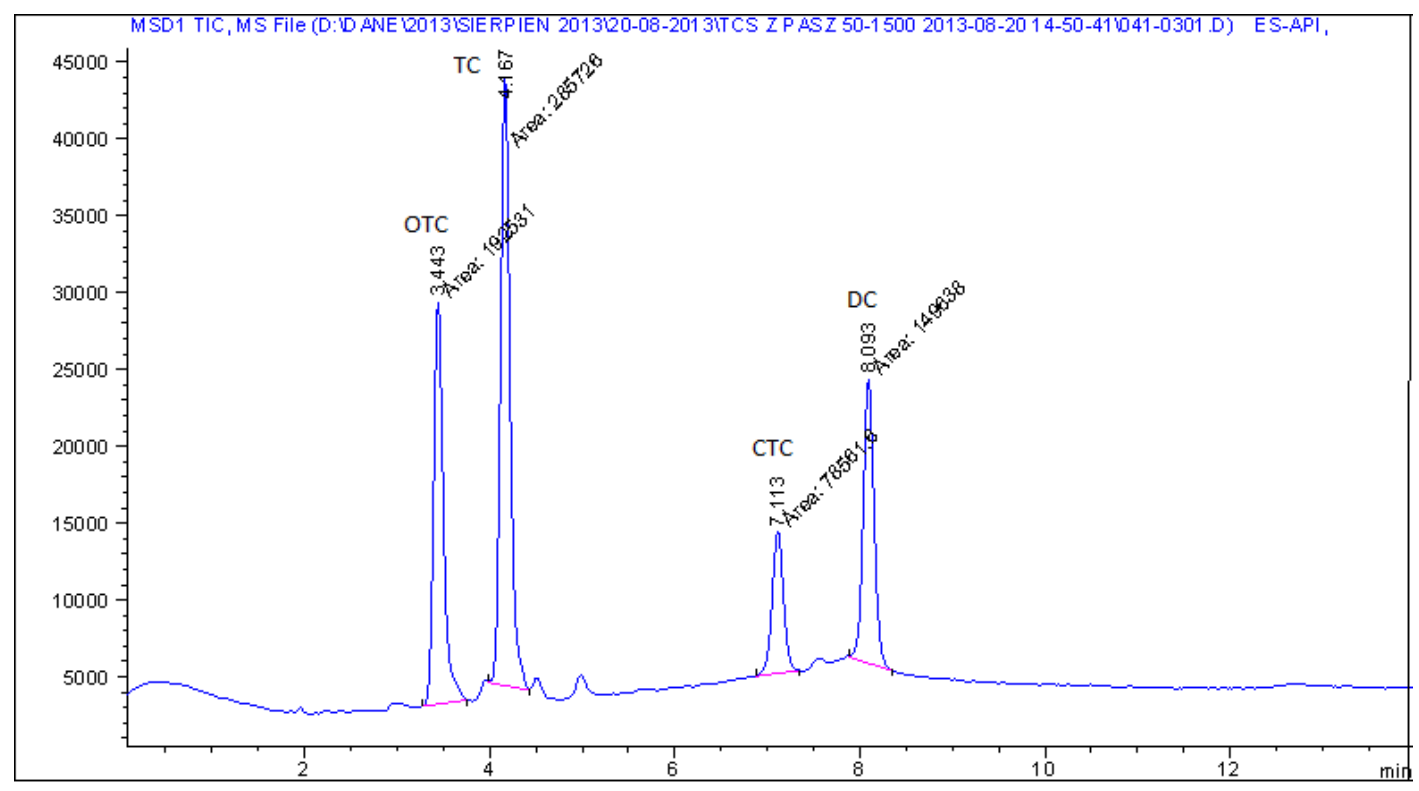

Fig. 6. LC-MS chromatogram of OTC, TC, CTC, and DC on a Zorbax Eclipse XDB C18 column with a mobile phase: $0.1 \%$ formic acid in $\mathrm{ACN} / 0.1 \%$ formic acid in water

Table 1. Recoveries, recoveries precision, and CVs of TCs in feeds

\begin{tabular}{|c|c|c|c|c|c|}
\hline \multirow[b]{3}{*}{ Analyte } & \multirow[b]{3}{*}{$\begin{array}{l}\text { Spiked } \\
\text { concentration } \\
(\mathrm{mg} / \mathrm{kg})\end{array}$} & \multicolumn{4}{|c|}{ Rates of recovery (\%) } \\
\hline & & \multicolumn{4}{|c|}{ Feed sample } \\
\hline & & $\begin{array}{l}\text { Recovery } \\
(\%) \\
(\mathrm{n}=18)\end{array}$ & $\begin{array}{l}\text { Intra-day } \\
\mathrm{CV}(\%) \\
(\mathrm{n}=6)\end{array}$ & $\begin{array}{l}\text { Inter-day } \\
\mathrm{CV}(\%) \\
(\mathrm{n}=18)\end{array}$ & $\begin{array}{l}\text { Recoveries } \\
\text { precision } \\
(\%)\end{array}$ \\
\hline \multirow{3}{*}{ Oxytetracycline } & 50 & 96.1 & 4.8 & 5.8 & \multirow{3}{*}{11.65} \\
\hline & 500 & 113.5 & 3.8 & 5.4 & \\
\hline & 1500 & 91.3 & 1.5 & 10.6 & \\
\hline \multirow{3}{*}{ Tetracycline } & 50 & 89.0 & 7.0 & 10.0 & \multirow{3}{*}{7.9} \\
\hline & 500 & 96.1 & 4.5 & 8.7 & \\
\hline & 1500 & 82.0 & 1.6 & 4.1 & \\
\hline \multirow{3}{*}{ Chlortetracycline } & 50 & 78.2 & 6.7 & 10.4 & \multirow{3}{*}{4.3} \\
\hline & 500 & 82.8 & 3.3 & 7.7 & \\
\hline & 1500 & 76.2 & 1.6 & 9.2 & \\
\hline \multirow{3}{*}{ Doxycycline } & 50 & 84.8 & 6.7 & 9.2 & \multirow{3}{*}{9.4} \\
\hline & 500 & 101.0 & 4.2 & 4.7 & \\
\hline & 1500 & 87.6 & 1.5 & 5.2 & \\
\hline
\end{tabular}

Table 2. LOD and LOQ values of tetracyclines in feeds

\begin{tabular}{lll}
\hline \multirow{2}{*}{ Analytes } & \multicolumn{2}{c}{ Feed sample } \\
\cline { 2 - 3 } & LOD,$(\mathrm{mg} / \mathrm{kg})$ & LOQ,$(\mathrm{mg} / \mathrm{kg})$ \\
\hline OTC & 4.2 & 5.7 \\
TC & 3.0 & 4.9 \\
CTC & 2.8 & 4.3 \\
DC & 3.2 & 4.9 \\
\hline
\end{tabular}

The repeatability and within-laboratory reproducibility for DC, OTC, CTC, and TC were lower than $9.0 \%$ for repeatability and $11 \%$ for withinlaboratory reproducibility at all spiked levels. The satisfactory results of precision expressed as coefficient of variation $(\mathrm{CV})$ showed that the presented methods can be used as validation methods and can be useful for confirmation of TC, OTC, CTC, and DC in medicated feedingstuffs. LOD and LOQ values for TCs in feeds ranged from 2.8 to 5.7 and from 4.3 to $5.7 \mathrm{mg} / \mathrm{kg}$ respectively (Table 2).

Specificity is the ability of a method to distinguish between the analyte of interest and other substances (impurities or matrix components) that may be present in a test sample. In the evaluation, the specificity of blank feed samples was analysed by MS detector. The 
results obtained with blank samples were compared with TC, CTC, OTC, and DC spiked samples and no interfering peaks were observed.

\section{Discussion}

The effective and efficient control of the declared value of the active substance, homogeneity, and appropriate therapeutic content, as well as monitoring of the stability of antimicrobial substances, led to development of method quantification using LC-MS.

The effective isolation of tetracyclines from feed matrixes is difficult because of their binding with sample proteins and chelating with metal ions. In numerous studies on the determination of tetracyclines, an extraction by the use of McIlvaine buffer solution with EDTA ( $\mathrm{pH} \mathrm{4)} \mathrm{has} \mathrm{been} \mathrm{described.} \mathrm{Aqueous-based}$ systems provide greater solubility for TCs than many organic compounds, excluding alcohols, and are miscible with the biological matrices of interest (1). The literature describes several methods of extraction of tetracycline antibiotics from feed matrices by means of extraction solutions, such as: methanol and $\mathrm{HCl}$, citric buffer with acetonitrile, water and methanol mixture, phosphoric buffer, acetonitrile, methanol and $1 \%$ formic acid mixture, and McIlvaine buffer-EDTA (pH 2 or 4$)(3,4,9,10-12,16,17,20)$.

The extracts of feeds are contaminated to a large extent, and therefore require further preparation of the sample. For this purpose different methods of purification of the extracts were tested in order to effectively eliminate endogenous substances which are co-extracted from the feed matrix and interfere with the analysis of tetracyclines. In the case of tetracycline treatment with animal medicated feedingstuff samples, in which these compounds are present in high concentrations (typically from 100 to up to $1000 \mathrm{mg} / \mathrm{kg}$ feed), purification of the extract is usually achieved by using only a membrane filter, usually a nylon filter with a pore size of 0.22 or $0.45 \mu \mathrm{m}$, or dilution of the extract with deionised water. In this work, mixtures of acetonitrile or methanol and McIlvaine-Na $\mathrm{NaDTA}_{2}$ buffer ( $\mathrm{pH} 4$ ) were used to compose alternative extraction solutions. The samples were spiked as indicated in the extraction and cleaned up with solutions using 10,100 , and $300 \mu \mathrm{g} / \mathrm{mL}$ of TC, OTC, CTC, and DC. The concentration of these compounds in the samples was 50, 500, and $1500 \mathrm{mg} / \mathrm{kg}$. The TC, OTC, CTC, and DC spiked samples were extracted with $10 \mathrm{~mL}$ aliquots of acetonitrile/buffer mixtures or methanol/buffer mixtures at $\mathrm{pH} 4$. The mixture of methanol and McIlvaine-Na ${ }_{2}$ EDTA $\mathrm{pH} 4$ in a ratio 6:4 $(\mathrm{v} / \mathrm{v})$ was selected as the useful combination for the procedure due to better recovery of active substances from feed samples. The extracts were diluted in deionised water and eluted with $0.1 \%$ formic acid in water and $0.1 \%$ formic acid in acetonitrile. The recoveries were calculated with respect to aqueous standard solutions injected and analysed in the same way.

An important part of each chromatographic separation is to choose appropriate analytical columns, suitable for the particular group of compound packings. For the separation of tetracycline antibiotics, C18 and C8 analytical columns are most commonly used $(4,6-$ 8, 16, 17, 20). PLRP-S polymeric columns (2, 13), Discovery Amide C18 (24), or Novopak phenyl column are used less frequently (18). Column lengths of either ca. $150 \mathrm{~mm}$ or $250 \mathrm{~mm}$ were equally popular, but a packing of $5 \mathrm{~mm}$ and column ID of ca. $4 \mathrm{~mm}$ were the most common (1).

Interaction of TCs with the silanols and trace metals present in silica packing materials significantly contributes to peak tailing and is often reported to be a problem during TCs chromatography. Therefore, it is important to choose a proper mobile phase. To avoid the formation of complexes with metal ions, and combining with the silanol groups on the columns of reversed phase (RP), the following most relevant components of the mobile phase have been described: formic acid, phosphate buffer, acetate buffer, citric acid and a mixture of butanol, sodium dodecyl sulfate, and acetic acid, o-phosphoric acid, perchloric acid, trifluoroacetic acid, or tetrahydrofurane and oxalic acid in water $(1,5)$. Column washing with an ethylenediaminetetraacetic acid (EDTA) solution prior to use is reported to remove the peak tailing due to metal impurities permanently. The acid acts as a simple ionisation suppression agent to minimize the occurrence of mixed separation mechanisms. Therefore, formic acid is often used for LC-MS technique. However, the most common acid used is oxalic acid, due to its additional ability to mitigate very effectively the effect of residual silanols on the stationary phase, and perhaps even to eliminate residual metals $(5,10,25)$. In this study, the best separation results were achieved on a Zorbax Eclipse XDB C18 column with the use $0.1 \%$ formic acid in water and $0.1 \%$ formic acid in acetonitrile. The use of gradient elution gave a much better separation of the analysed compounds. The developed method gave good results for both samples prepared in-house (spiked with the appropriate levels of OTC, TC, CTC, and DC) and those delivered by manufacturers.

The procedure can be applied successfully to the rapid and sensitive analysis of TCs in animal medicated feedingstuffs using reserved-phase liquid chromatography with ESI-MS and gradient elution. The extraction of the antibiotics is based on a simple extraction step, and no clean-up steps are necessary. The presented LC-MS method is efficient, precise, and useful for routine analysis. The presented results proved the suitability of the method for the use in testing medicated feedingstuffs quality and homogeneity, and quantitative determination of active substances in the feeds. 
Conflict of Interests Statement: The authors declare that there is no conflict of interests regarding the publication of this article.

Financial Disclosure Statement: The study was financially supported by the National Veterinary Research Institute, Pulawy, Poland.

\section{Animal Rights Statement: None required.}

\section{References}

1. Andersen W.C., Roybal J.E., Gonzales S.A., Turnipseed S.B., Pfenning A.P., Kuck L.R.: Determination of tetracycline residues in shrimp and whole milk using liquid chromatography with ultraviolet detection and residue confirmation by mass spectrometry. Anal Chim Acta 2004, 529, 145-150.

2. Blanchflower W.J., McCracken R.J., Rice D.A.: Determination of chlortetracycline residues in tissues using high-performance liquid chromatography with fluorescence detection. Analyst 1989, 114, 421-423.

3. Boscher A., Guignard C., Pellet T., Hoffman L., Bohn T.: Development of a multi-class method for the quantification of veterinary drug residue in feedingstuffs by liquid chromatography-tandem mass spectrometry. J Chromatogr A 2010, 1217, 6394-6404.

4. Caballero R.D., Torre-Lapasio R., Garcia-Alvarez-Coque M.C., Ramis-Ramos G.: Rapid liquid determination of tetracyclines in animal feeds using a surfactant solution as mobile phase. Anal Lett 2002, 35, 687-705.

5. Carrasco-Pancorbo A., Casado-Terrone S., Segura-Carretero A., Fernández-Gutiérrez A.: A reversed-phase high-performance liquid chromatography coupled to ultraviolet and electrospray time-of-flight mass spectrometry on-line detection for the separation of eight tetracyclines in honey samples. J Chromatogr A 2008, 1195, 107-116.

6. Chafer-Pericas C., Maquieira A., Puchades R., Miralles J., Moreno A.: Multiresidue determination of antibiotics in feed and fish samples for food safety evaluation. Comparison of immunoassay vs LC-MS-MS, Food Control 2011, 22, 993-999.

7. Cherlet M., Schelkens M., Croubels S., De Backer P.: Quantitative multi-residue analysis of tetracyclines and their 4-epimers in pig tissues by high-performance liquid chromatography combined with positive-ion electrospray ionization mass spectrometry. Anal Chim Acta 2003, 492, 199-213.

8. Fiori J., Grassigli G., Filippi P., Gotti R., Cavrini V.: HPLCDAD and LC-MS analysis of doxycycline and related impurities in doxipin mix, a medicated premix for incorporation in medicated feedstuffs. J Pharma Biomed Anal 2005, 37, 979-985.

9. Gafner J.L.: Identification and semiquantitative estimation of antibiotics added to complete feeds premixes, and concentrates. J AOAC Int 1999, 82, 1-8.

10. Guo L., Chen Y., Zhang L., Yang W., He P.: Development and validation of a liquid chromatographic/tandem mass spectrometric method for determination of chlortetracyclines, oxytetracyclines, tetetracyclines, and doxycyclines in animal feeds. J AOAC Int 2012, 95, 1010-1015.

11. Injac R., Mlinaric A., Djorjevic-Milic V., Karljikovic-Rajic K., Strukelj B.: Optimal conditions for determination of zinc bacitracin, polymyxin B, oxytetracycline and sulfacetamide in animal feed by micellar electrokinetic capillary chromatography. Food Addit Cont 2008, 4, 424-431.

12. Kaklamanos G., Vincent U., von Holst C.: Analysis of antimicrobial agents in pig feed by liquid chromatography coupled to orbitrap mass spectrometry. J Chromatogr A 2013, 1293, 60-74.

13. Khan N.H., Roets E., Hoogmartens J., Vanderhaeghe H.: Quantitative analysis of oxytetracycline and related substances by high-performance liquid chromatography. J Chromatogr A 1987, 405, 229-245.

14. Ma T.Y., Vickroy T.W., Shien J.H., Chou C.C.: Improved nonaqueous capillary electrophoresis for tetracyclines at subparts per billion level. Electrophoresis 2012, 33, 1679-1682.

15. Markaksis P.K.: Determination of tetracyclines in animal feeds in the presence of other drugs by thin-layer chromatography and microbiological method. J AOAC Int 1996, 79, 375-379.

16. Martinez E., Shimoda W.: Liquid chromatographic determination of tetracycline residue in animal feeds. J Assoc Off Anal Chim 1987, 71, 477-480.

17. Martinez E., Shimoda W.: Liquid chromatographic determination of epimerization of chlortetracycline residue to 4-epi-chlorotetracycline residue in animal feed, using McIlvain's buffer as extractant. J Assoc Off Anal Chem 1989, 72, 848-850.

18. Mulders E.J, Van De Lagemaat D.: Determination of residues of tetracycline antibiotics in animal tissues by high-performance liquid chromatography. J Pharm Biomed Anal 1992, 7, 1829-1835.

19. Oka H., Ito Y., Matsumoto H.: Chromatographic analysis of tetracycline antibiotics in foods. J Chromatogr A 2000, 882, 109-133.

20. Patyra E., Kowalczyk E., Kwiatek K.: Development and validation method for the determination of delected tetracylines in animal medicated feedingstuffs with the use of micellar liquid chromatography. Anal Bioanal Chem 2013, 405, 6799-6806.

21. Schnappinger D., Hillen W.: Tetracyclines: antibiotic action, uptake, and resistance mechanisms. Arch Microbiol 1996, 165, 359-369.

22. Tong J., Rao Q. K., Jiang Z., Ding S.: Simultaneous determination of five tetracycline and macrolide antibiotics in feed using HPCE. J Sep Sci 2009, 32, 4254-4260.

23. Udalova A.Y., Dmitrienko S.G., Apyari V.V.: Methods for the separation, preconcentration, and determination of tetracycline antibiotics. J Anal Chem 2015, 70, 661-676.

24. Vinas P., Balsalobre N., Lopez-Erroz C., Hernandez-Cordoba M.: Liquid chromatography with ultraviolet absorbance detection for the analysis of tetracycline residue in honey. J Chromatogr A 2004, 1022, 125-129.

25. Walsh J., Walker L.V., Webber J.J.: Determination of tetracyclines in bovine and porcine muscle by high-performance liquid chromatography using solid-phase extraction. J Chromatogr A 1992, 596, 211-216. 